East African Medical Journal Vol. 84 No. 9 September 2007

RHEUMATIC DISEASES IN PATIENTS ATTENDING A CLINIC AT A REFERRAL HOSPITAL IN YAOUNDE, CAMEROON

M. Singwe-Ngandeu, MD, Senior Lecturer, Rheumatologist, Internal Medicine Unit, General Hospital, P.O. Box 5408, Yaounde, Cameroon, J. Meli, MD, Senior Lecturer, Epidemiologist, Department of Public Health, Faculty of Medicine and Biomedical Sciences, University of Yaounde, Cameroon, H. Ntsiba, MD, Associate Professor, Rheumatologist, Rheumatology Department of Congo-Brazzaville University Teaching Hospital, C. Nouédoui, MD, Associate Professor, Endocronologist, A.V. Yollo, MD, General Practitioner, M.B.Sida, MD, Associate Professor, Hepato-gastro-enterologist, and W.F. Muna, MD, Professor, Cardiologist, Internal Medicine Unit, General Hospital Yaounde, P.O. Box 5408, Yaounde, Cameroon

Request for reprints to: Dr. M. Singwe-Ngandeu, Internal Medicine Unit, General Hospital Yaounde P.O. Box 5408 Yaounde, Cameroon

\title{
RHEUMATIC DISEASES IN PATIENTS ATTENDING A CLINIC AT A REFERRAL HOSPITAL IN YAOUNDE, CAMEROON
}

\author{
M. SINGWE-NGANDEU, J. MELI, H. NTSIBA, C. NOUÉDOUI, A.V. YOLLO, M.B. SIDA and \\ W.F. MUNA
}

\begin{abstract}
Background: The spectrum of rheumatic diseases in sub-Saharan Africa remains poorly defined. Objectives: To determine the spectrum of rheumatic diseases in Yaounde.

Design: Descriptive cross-sectional study.

Setting: Rheumatology clinic at the Yaounde General Hospital, Cameroon.

Subjects: Twelve thousand four hundred and ninety four patients were referred to the outpatient department of the Internal Medicine service of the General Hospital of Yaounde over a 12-month period. Of these cases, $536(9.4 \%)$ were diagnosed as belonging to the general class of rheumatic conditions. There were $334(62.31 \%)$ females and $202(37.69 \%)$ males. The mean age was $52.72 \pm$ 5.3 years.

Results: The 536 cases were further classified as follows: degenerative disease of the spine 196 (36.5\%), osteoarthritis of the limbs 110 (20.5\%), regional musculo-skeletal disorders $83(15.5 \%)$, arthritis associated with infections $50(9.3 \%)$, chronic inflammatory and connective tissue diseases $44(8.2 \%)$, crystalline arthropathies $32(5.9 \%)$ and miscellaneous (unclassified) rheumatic conditions $21(4.1 \%)$.

Conclusion: From these results, we concluded that degenerative disease of the spine and limbs are the most common forms of rheumatic disease in this region. Gout is the most commnon acute inflammatory arthropathy and rheumatoid arthritis the most prevalent arthropathy due to chronic inflammation.
\end{abstract}

\section{INTRODUCTION}

Some recent observations (1-4) have suggested that rheumatic conditions are increasingly becoming an important cause of morbidity and mortality in sub-Saharan Africa (SSA) despite the current focus on other important causes such as infectious diseases (especially HIV/AIDS and malaria) and malnutrition. In addition, the period extending from $2000-2010$ has been declared 'the bone and joint' decade by the World Health Organization (WHO). This declaration was intended to attract world attention to the disability due to musculoskeletal disorders and to ensure that appropriate care is available to all populations in need. There are very few publications and no reliable data on the prevalence of the various rheumatic conditions in the SSA region. Not much is known about the exact 
natural history and risk factors for these conditions within the SSA populations. The current context of increasing poverty and diminishing allocations despite an increasing number of competing healthcare issues constitutes a constant challenge for governments in SSA. Given the growing importance of these disease entities, such information will be necessary in conceiving and establishing preventive programmes. It may also prove to be critical in the determination of healthcare needs and costs in addition to providing a rational component for developing overall healthcare planning.

The purpose of this preliminary study was to establish the spectrum of rheumatic diseases among adult patients attending an outpatient department of a tertiary healthcare facility in Cameroon.

\section{MATERIALS AND METHODS}

Cameroon is located within the central African region between latitudes $2^{\circ}$ and $13^{\circ}$ north with a surface of $475,442 \mathrm{~km}^{2}$ and a population estimated to be around $16,000,000$. Yaounde has a population of $1,400,000$ and it's the capital city of Cameroon and one of several urban centers. Yaounde General Hospital is one of six public referral tertiary health care facilities in Cameroon. This facility alone receives about 45,000 out-patients per year. A rheumatology unit was established within the Internal Medicine service of the hospital in October 2000. The unit has only one rheumatologist. Available imaging techniques include plain roentgenograms, ultrasound and CT-Scan. Laboratory capabilities include those for routine tests such as blood count, thick smear to search for blood parasites, cultures and erythrocytes sedimentation rate. Other analytic techniques including Elisa for HIV, rheumatoid factor, hepatitis $B$ and $C$ antigen and antibody are also available.

This cross-sectional study was carried out from October 2000 to October 2001. Only individuals 16 years of age or older at the time of their first visit to the hospital, were included in the study. A complete history and physical examination with emphasis on signs and symptoms of rheumatic conditions was performed on each patient. Basic demographic information and anthropometrical measures were also obtained for each patient. A complete blood count and erythrocytes sedimentation rate were performed in all patients. When strongly suggested by elements from the history, clinical examination and other investigations, rheumatoid factor, antinucleic antibodies, anti doubled stranded DNA, anti SCL 70, anti topoisomerase antibodies, antiphospholipid antibodies and HIV serology were performed. Plain roentgenograms were obtained for swollen, painful and inflamed joints and bones. In cases where there was clinical evidence of a joint effusion, arthrocenthesis was performed to allow for cytological and bacteriological analysis as well as a search for crystals. Computerized axial tomography (CT scan) and sonography as well as in cases where it was thought to be useful in establishing a more accurate diagnosis. Tomodensitometry was also performed in few cases when necessary. Several investigations such as bone or synovial biopsy, scintigraphy, magnetic resonance imaging, osteodensitometry, HLA typing, cryoglobulinemia and a search for ANCA (antineutrophil cytoplasme antibodies) were not conducted in our patients for lack of the necessary infrastructure.

The rheumatic disorders were diagnosed and classified according to the International Diseases Classification criteria (5).

\section{RESULTS}

Twelve thousand four hundred and ninety four outpatient consultations were recorded during the one-year period in internal medicine department of the General Hospital of Yaounde. Among them, 536 (9.4\%) patients with rheumatic diseases were identified. There were $334(62.31 \%)$ females and $202(37.69 \%)$ males. The mean age was $52.72 \pm 5.3$ years. The diagnosis, age distribution (mean age and standard deviation), and sex ratio (female/male) of patients presenting with rheumatic diseases are shown in Table 1 . The mean body mass index was $26.6 \mathrm{~kg} / \mathrm{m}^{2}$.

Mechanical disorders of the spine occurred in $196(36.5 \%)$ of the 536 patients (Table 2). The mean age of patients suffering from these conditions was $50.82 \pm 10.15$ years. One hundred and twenty nine (65.8\%) of these 196 patients complaining of either neck or low back pain, were women. Low back pain was found in $160(81.63 \%)$ patients and was as followed by lumbago: seven (3.8\%) cases; chronic low back pain 86 (43.8\%) cases; low back pain with sciatica $67(34.2 \%)$ cases. Dorsal pain was noted in nine $(4.66 \%)$ cases. Neck pain occurred in 23 $(11.73 \%)$ of the 196 patients. Four of these 23 patients presented with cervical radiculopathy. 
Table 1

Distribution of the various rheumatic entities according to age, sex ratio and disease category

\begin{tabular}{lrrr}
\hline Condition & No. & $(\%)$ & Sex ratio (F/M) \\
\hline Mechanical disorders of the spine & 196 & 36.49 & $129 / 67$ \\
Osteoarthritis & 110 & 20.48 & $76 / 34$ \\
Regional musculoskeletal disorders & 83 & 15.45 & $50 / 33$ \\
Arthritis associated with infectious agents & 50 & 9.32 & $29 / 21$ \\
Chronic rheumatic inflammatory and systemic connective & 44 & 8.19 & $30 / 14$ \\
$\quad$ tissue diseases & 32 & 5.95 & $3 / 29$ \\
Crystal-related arthropathies & 21 & 4.09 & $15 / 6$ \\
Miscellaneous rheumatic diseases & 536 & 100 & $334 / 202$ \\
\hline Total & & & \\
\hline
\end{tabular}

Table 2

Mechanical disorders of the spine

\begin{tabular}{llrc}
\hline & Diseases & Frequency & $(\%)$ \\
\hline \multirow{2}{*}{ Low back pain } & Lumbago & 7 & 3.7 \\
& Chronic low back pain & 86 & 43.8 \\
& Low back with sciatica & 67 & 34.2 \\
Dorsal pain & & 9 & 4.6 \\
Neck pain & Acute neck pain & 2 & 1 \\
& Chronic neck pain & 21 & 10.7 \\
& Neck pain with & 4 & 2 \\
& radiculopathy & & \\
\hline Total & & 196 & 100 \\
\hline
\end{tabular}

Osteoarthritis of the limbs was found in 110 (20.5\%) of 536 patients diagnosed with rheumatic conditions. The knee joint was involved in $87(79.1 \%)$ of the 110 patients with a diagnosis of osteoarthritis. Ten $(9.1 \%)$ patients had hip joint involvement. The other involved joints included shoulder: three $(2.72 \%)$ patients; metacarpo-phalangeal joint: three $(2.72 \%)$ patients; ankle joint: three $(2.72 \%)$ patients; tarsal joint: three patients $(2.72 \%)$; and wrist joint in one $(0.92 \%)$ patient (Table 3$)$.

A diagnosis of regional musculo-skeletal disorders was made in $83(15.5 \%)$ of the 536 cases. The shoulder joint was involved in $32(38.6 \%)$ of the 83 cases. The following joints were also involved: the hip joint $24(28.9 \%)$ cases; the plantar fasceitis joint $11(13.3 \%)$ patients; trigger finger deformity in nine $(10.8 \%)$ patients; De Quervain's tenosynovitis in three $(3.6 \%)$ patients; bursitis in two $(2.4 \%)$ patients and the carpal tunnel syndrome in two (2.4\%) patients.

Fifty $(9.3 \%)$ of the 536 patients presented with arthritis in the context of an infectious agent. Rheumatic syndromes in $31(62 \%)$ of the 50 patients were thought to be related to the HIV infection confirmed by positive serology. These cases were classified as AIDS-associated rheumatic syndromes. The other cases of arthritis thought to be related to ongoing infections were classified as follows; eight $(16 \%)$ cases of parasitic infestation diagnosed in patients with microfilaria Loa Loa; six (12\%) cases in hepatitis $\mathrm{C}$ infection; four ( $8 \%$ ) cases of pyogenic arthritis in patients with sepsis and one $(2 \%)$ case of Pott's disease. 
Arthropathies due to crystal formation within the joint space were identified in $32(5.9 \%)$ of the 536 patients. Gout was the unique diagnosis.

Table 3

Different locations of osteoarthritis of the limbs

\begin{tabular}{lcc}
\hline Joint & Frequency & $(\%)$ \\
\hline Knee & 87 & 79.1 \\
Hip & 10 & 9.1 \\
Metacarpo-phalangeal joint & 3 & 2.72 \\
Shoulder & 3 & 2.72 \\
Ankle & 3 & 2.72 \\
Tarsal & 3 & 2.72 \\
Wrist & 1 & 0.92 \\
\hline Total & 110 & 100 \\
\hline
\end{tabular}

Chronic rheumatic inflammatory and systemic connective tissue diseases were identified in 44 (8.2\%) (Table 4). The type of arthritis could not be classified in $15(34.1 \%)$ in instances. There were $13(29.5 \%)$ cases of rheumatoid arthritis and four $(9.1 \%)$ cases of ankylosing spondylitis. Systemic lupus ery thematosis was found in four $(9.1 \%)$ cases, acute rheumatic fever in three $(6.81 \%)$ cases, progressive systemic sclerosis in two $(4.5 \%)$ cases, polymyositis in one $(2.3 \%)$ patient, juvenile idiopathic arthritis one $(2.3 \%)$ patient and psoriatic arthritis in one $(2.3 \%)$ patient.

\section{Table 4}

Chronic rheumatic inflammatory and systemic connective tissue diseases

\begin{tabular}{lcc}
\hline Disease & Frequency & $(\%)$ \\
\hline Unclassified arthritis & 15 & 34.1 \\
Rheumatoid arthritis & 13 & 29.5 \\
Systemic lupus erythematosus & 4 & 9.1 \\
Ankylosing spondylitis & 4 & 9.1 \\
Acute rheumatic fever & 3 & 6.8 \\
Systemic sclerosis & 2 & 4.5 \\
Psoriatic arthritis & 1 & 2.3 \\
Polymyositis & 1 & 2.3 \\
Juvenile idiopathic arthritis & 1 & 2.3 \\
Total & 44 & 100 \\
\hline
\end{tabular}

Twenty one $(4.1 \%)$ of the 536 patients with rheumatic conditions were classified in a miscellaneous group. Eleven $(52.4 \%)$ of these
21 patients had joint pain of unknown origin, two $(9.5 \%)$ had fibromyalgia, two $(9.5 \%)$ had sarcoidosis, one (4.8\%) had multiple myeloma, one (4.8\%) had Sudeck's atrophy, and one (4.8\%) had pyomyositis. A single patient was also registered with each of the following conditions: rhabdomyoma, hepatocarcinoma with metastatic bone lesions, achondroplasia and chondromalacia of the patella.

\section{DISCUSSION}

This study describes the spectrum of rheumatic diseases seen at the outpatient department of a referral and tertiary care hospital in Cameroon. The study has raised several issues ranging from the design to the conclusions that could be drawn from the results. Firstly, the study represents a highly selected population with a strong recruitment bias. It is in no way intended to reflect the freeliving population pattern of disease in Cameroon. Secondly, there are few trained rheumatologists in SSA in general and in Cameroon in particular. This has resulted in a high level of centralization of both diagnostic and therapeutic resources, whether they are related to infrastructure, technology or human capacity. Such a degree of centralization of case-referrals can permit the collection of a wider spectrum of patients from rural and urban Cameroon. Thirdly, Cameroon is unique in its central geographic location within the African continent around the equator and it extends over several vegetation and climatic zones from tropical, subtropical, savannah, semi-arid and arid desert regions. This location can permit a better insight into the relationships between disease patterns and climatic conditions within the SSA region.

Rheumatic conditions contribute significantly to the disease burden among patients in the General Hospital of Yaounde in Cameroon. We found that $9.4 \%$ of all outpatients in internal medicine were suffering from rheumatic diseases. The importance of the $10 \%$ frequency of rheumatic diseases could become more important and be better appreciated if it were to be expressed in disease burden units as 'DALY's' (or disability-adjusted life years). Our findings concur with the data from similar settings in sub-Saharan Africa (2,3,6-8). In this study, mechanical lesions of the spine were the basis of the most prevalent complain of low back pain from patients presenting at our clinic. Low back 
pain and sciatica were relatively common. Obese women ( $66 \%$ of the women consulting) either from rural or urban backgrounds and regardless of their occupations appeared to be particularly at risk. Similar observations have been reported from the Republic of Togo (8). Degenerative diseases (with no specific aetiology, pattern or distribution) of the spine were the most common lesions followed by osteoarthritis of the knee.

The high prevalence of gonarthrosis in black Africa has been highlighted in other reports (2-4). Such prevalence contrasts with the very low prevalence of coxarthrosis (only 12\% cases observed). A similarly low prevalence has also been reported in Togo (2). We observed only one case of secondary coxarthrosis in a young female patient suffering from a congenital dysplasia of both hip joints. Contrary to other reports from West Africa (2), no case of haemoglobinopathy was diagnosed in the patients reported here. We noted other localisations of degenerative osteoarthritis (thought to be secondary in each case): shoulder, tarsus, ankles, metacarpo-phalangeal and wrist joints. Regional musculoskeletal disorders consisted mainly of shoulder and bicipital tendinitis, trigger finger and plantar fasciitis (usually associated with flat foot).

We suspect that HIV infection, which is endemic in SSA, is responsible for a considerable number of arthropathies. These cases of arthritis are predominantly asymmetrical, monoarticular or polyarticular in nature, affecting both small and big joints as reported by Bileckot et al (9). Our experience here in Cameroon suggests similar findings among our HIV-positive patients.

We observed a relatively large number (compared to other studies conducted in Africa) arthropathies associated with parasitic infections. Micro filaria Loa Loa was the culprit in seven of the eight cases of parasitic arthropathies observed in the current study. These patients presented with polyarthralgia, Calabar swelling associated with pruritus and very often located on the back of the hand, the forearm or dorsum of the foot. The patients presented no superficial or systemic signs of inflammation. In one case, the presence of live filarial ( Loa Loa) worms was observed in the conjunctivae. Hypereosinophilia was present in all patients with the filarial infection. The clinical status of all patients improved with the administration of adapted antifilaria treatment.

Six cases of arthropathy related to presence of hepatitis C were observed. Screening for cryoglobulins was not carried out in any of these patients. Previous studies conducted in the SSA region did not mention these arthropathies (2-4).

The cases of septic arthritis and spondylodiscitis observed were similar typical of previously described cases (3). We observed only one case of Pott's disease. This may be somewhat of an unexpected finding given the recrudescence of tuberculosis today in the SSA region. Alternately, such patients could have been referred to specialized tuberculosis clinics and treated appropriately because of obvious primary lesions in common sites such as the lung.

With respect to the observed cases of inflammatory arthropathies, gout was the most common condition. This observation is consistent with previously published reports from the SSA region $(2,3,10)$. In fact, gout is more common than all the other inflammatory arthropathies combined. In addition, the clinical pattern (including other parameters such as average age and sex) observed in the patients with gout in this report is similar and consistent with other published reports from the SSA region (11).

Of the 44 patients observed with chroaic inflammatory arthropathies more than a quarter o: 13 (29.5\%) met the American College of Rheumatology (ACR) criteria for the diagnosis of rheumatoid arthritis (12), while four (9.1\%) observed cases of spondyloarthropathy met the diagnostic criteria suggested by Amor et al (13). These results are in contrast with findings by Mijiyawa et al (2), who reported a very low prevalence of rheumatoid arthritis: only two $(0.2 \%)$ cases were found. We also describe four $(9.1 \%)$ cases of systemic lupus erythematosis who presented with primary polyarthritis, pericarditis, malar rash and in whom we found antinuclear antibodies as well as antibodies against double stranded DNA (14). Two patients met ACR criteria for the diagnosis of systemic sclerosis (15). In $15(34.1 \%)$ or about one third of the 44 of the cases of inflammatory arthropathies, a specific disease entity could not be assigned and thus were classified as arthritis of unknown origin. This inability to assign some of these patients to specific disease groups was due to the unavailability in their hospital records of essential and specific laboratory tests required for diagnosis.

Finally, we observed a miscellaneous group of rheumatic diseases which included four cases of polyarthralgia without clinical evidence of synovitis. 
Two cases of fibromyalgia were observed in two young native Cameroonian women, living in a rural environment since birth. It is equally interesting to note that they had clinical presentation identical to that described in western population groups of developed countries (16).

In conclusion, the characterisation and accurate diagnosis of rheumatic conditions in the SSA region is becoming more and more feasible because of the increasing availability of specific technology in medical imagery as well as biochemical and immunologic tests. This highly selective hospitalbased study suggests that rheumatic diseases may constitute an important source of disease burden in the SSA region. About $10 \%$ of outpatient referrals to a tertiary care facility in Yaounde Cameroon could be attributed to one of several common rheumatic diseases. Degenerative arthropathies of the spine and the limbs were the most prevalent conditions encountered. The implication of this finding for the future development of preventive and therapeutic strategies cannot be overestimated, although the identification of risk factors (obesity and other dietary factors, occupation as well as other lifestyle modification factors) was not a primary objective of this study. Gout was a commonly encountered crystalline arthropathy and rheumatoid arthritis was the most prevalent chronic inflammatory rheumatic disease. A more complete understanding of the impact of rheumatic diseases in this region will require further studies of this type as well as free-living population-based studies. It will even be more important to identify risk factors whether they be related to a particular lifestyle such as diet, occupation as well as other factors related to the climate or environment. A more complete description and understanding of genetic as well as other biologic determinants will be equally important.

\section{ACKNOWLEDGEMENT}

To all patients who accepted to participate in this study.

\section{REFERENCES}

1. Adebajo A.O. and Davis P. Rheumatic diseases in African blacks. Semin. Arthritis Rheum. 1994; 24: 139-153.
2. Mijiyawa M.A., Djagnikpo A.K., Agbanouvi A.E., Koumouvi K. and Agbetra A. Maladies rhumatismales observées en consultation hospitalière à Lomé (Togo). Rev. Rhum. Mal. Ostéoartic. 1991; 58: 349-354.

3. Bileckot R., Ntsiba H., Mbongo J.A., Masson Ch. and Brégeon $\mathrm{Ch}$. Les affections rhumatologiques observées en milieu hospitalier au Congo. Sem. Hôp. Paris. 1992; 68: 282-285.

4. Stein M. and Davis P. Rheumatic disorders in Zimbabwe: A prospective analysis of patients attending a rheumatic disease clinic. Ann. Rheum. Dis. 1990; 49: 400-402.

5. Classification Internationale des maladies. Genève: Organisation Mondiale de la santé 1977.

6. Oyoo G.O. Rheumatic disorders in Kenya. I. Spectrum of disease (abstract). Clin. Rheumatol. 2007; 26: 43

7. Adebajo A.O. Pattern of osteoarthritis in west african teaching hospital. Ann. Rheum. Dis. 1991; 50: 20-22.

8. Mijiyawa M., Koumouvi K., Bellow A., N'dakena K.and Doury P. Pathologies dégénératives du rachis en consultation hospitalière à Lomé (Togo). Rev. Rhum. Mal. Ostéoartic. 1992; 59: 797-800.

9. Bileckot R., Mouaya A. and Makuwa M. Prevalence and clinical presentations of arthritis in HIV-positive patients seen at a rheumatology department in CongoBrassaville. Rev. Rhum. (Engl. Ed.). 1998: 65: 549-554.

10. Jeandel P., Sanga M., Fankam H. and Kouda Zeh A. Les arthropathies inflammatoires au Cameroun. Etude prospective de 112 cas. Med. Afr. Noire. 1988; 35: $836-845$

11. Mijiyawa M., Djagnikpo K., Dagbovie K. and Agbetra A. La goutte chez les malades togolais. Rev. Rhum. Mal. Ostéoartic. 1992; 59: 473- 477.

12. Arnette F.C., Edworthy SM., Bloch D.A., et al. The American Rheumatism Association 1987 revised criteria for the classification of rheumatoid arthritis. Arthritis Rheum. 1988; 31: 315-324.

13. Amor B., Dougados M.A. and Mijiyawa M. Critères de classification des spondylarthropathies. Rev. Rhum. Mal. Ostéoartic. 1990; 57: 85-89.

14. Tan E.M., Cohen A.S., Fries J.F., et al. The 1982 revised criteria for classification of systemic lupus erythematosus. Arthritis Rheum. 1982; 1271-1277.

15. be Roy E.C., Black C., Fleischmajer R., et al. Scleroderma (systemic sclerosis): Classification, subsets and pathogenesis. J. Rheumatol. 1998; 15: 202-205.

16. Wolfe F., Smythe H.A., Yunus M.B., Bennett R.M., et al. The American College of Rheumatology 1990 criteria of the classification of fibromyalgia. Arthritis Rheum. 1990; 33: 160-172. 